\title{
Ferroelasticity comes of age
}

\section{Crystals that can be made to twin under the influence of mechanical stress have been known for some time; neodymium pentaphosphate may become the basis of novel optical and acoustic devices.}

WHAT is the object shown in the accompanying photograph? An optical grating perhaps? Then something has gone wrong with the ruling engine, for the rulings are not uniform in width but wedge-like, making a zig-zag pattern across each strip of the object. Yet potentially, the first guess is correct.

What the photograph shows is the pattern of transmission of polarized light through a single crystal of neodymium pentaphosphate (NPP) prepared by a technique described by Stephen Meeks and B.A. Auld in the $15 \mathrm{July}$ issue of Applied Physics Letters (47, 102; 1985). The width of the periodic bands ranges from roughly $18 \mu \mathrm{m}$ to $6 \mu \mathrm{m}$ (on the right).

What excites Meeks and Auld is that they have a technique by which the spacing of the grating elements can be systematically changed, or tuned, at least for spacings greater than $0.5 \mu \mathrm{m}$. Since NPP is also a laser material, and one with an exceptionally low threshold because of the exceptional fluorescence of the material, they hope to be able to have a laser with a built-in optical grating which can, moreover, be instantaneously tuned. But the material has other obvious applications in, for example, the construction of acoustic gratings and filters.

This curious development has been on the cards for the best part of a decade, since the recognition that the pentaphosphates of the rare earth elements (but also those of lead and bismuth) are capable of forming crystals that are ferroelastic, which is merely a way of saying that they may be induced to change their microscopic state by means of macroscopic elastic stress. Indeed, NPP crystals, which tend to be produced as thin plates that may be up to a centimetre long, can be flipped from one stable state to another by forces that seem quite trivial; stroking the surface of a crystal with a toothpick seems to suffice.

The explanation of this peculiar behaviour of NPP, and of materials like it, seems first to have been given by H.P. Weber, working at Bell Laboratories in the 1970 s (see, for example, Weber, H.P., Tofield, B.C. \& Liao, P.F. Phys. Rev. B11, 1152; 1975). The essence of the explanation is that NPP may crystallize in the form of monoclinic crystals which differ hardly at all from the higher orthorhombic symmetry. Indeed, X-ray diffraction shows NPP to have pseudoorthorhombic symmetry; numerically, the monoclinic angle differs from $90^{\circ}$ by roughly $1 / 2^{\circ}$ only.

The result is that parts of a crystal may be pushed, literally or mechanically, from one monoclinic form into the other lying the other side of the orthorhombic ideal. The result is a twinned crystal. The patterns that Meeks and Auld have produced at the Applied Physics Department at Stanford University are nothing but those

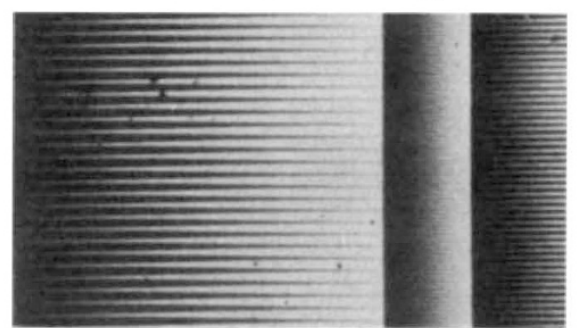

of crystals in which twinned structures alternate with each other across the width of the whole specimen.

All this is accounted for by the atomic structure of the crystals. NPP is $\mathrm{NdP}_{5} \mathrm{O}_{14}$, and the monoclinic forms of the material are built, on an atomic scale, from tetrahedra of $\mathrm{PO}_{4}$ ions linked together by the commonality of oxygen atoms.

The basic building-block of the crystal structure is a more or less flat ring of eight tetrahedra; these units are linked to other identical rings by means of pairs of phosphate tetrahedra. The result is a ribbon of linked phosphate tetrahedra some $13 \AA$ across and arbitrarily long. In the bulk crystals, which are cemented together by the appropriate disposition of $\mathrm{Nd}$ ions, the ribbons are laid down parallel to each other to form sheets, and then these sheets are piled on top of one another.

The origin of the ferroelasticity of NPP lies in the asymmetry of the eightmembered phosphate rings, which are so constructed as to bring one pair of oxygen atoms (on opposing tetrahedra) too close together for comfort. The result is a distortion of the ring from the most symmetrical arrangement that might be attained. The distortion is only small and, as might be expected, it disappears as the temperature is increased, which is why monoclinic NPP is transformed into rhombic NPP at a little above $140^{\circ} \mathrm{C}$.

The formation of twins in such a crystal is then easily accounted for. The distortion of the basic phosphate ring in the crystal structure can be accomplished either by moving the tip of one of a pair of opposing tetrahedra to the right and the other to the left, or vice versa. So one part of a monoclinic crystal may consist of rings distorted in one sense and the remaining rings distorted in the other. The horizontal stripes in the figure are such twins.

Because of the layered structure of NPP, however, it is clear that another form of twinning is possible, essentially because the registration of successive planes of phosphate ribbons can be changed relative to each other. Twinning of this kind, which is not in practice as easily accomplished, accounts for the vertical boundaries in the illustration.

The trick that Meeks and Auld now boast of is that for tuning the periodicity of the wedge-shaped alternating domains of crystal structure in twinned relationship to each other. Generating a pattern (such as that in the illustration) is largely a matter of supporting a plate-like crystal at two points and pressing between the supports.

The use of the word domain is deliberate; Meeks and Auld point out that the effects of a first application of stress are to cause the formation of a number of flat lens-shaped bubbles of different twinned crystal structure which, like ferromagnetic domains, appear to repel each other, so that their spacing is reasonably regular. The greater the shear stress on the crystal, the smaller the spacing. When the bubbles fill the space between the pressure points, they coalesce into a zig-zag pattern as in the figure, and when the stresses are removed, that part of the pattern outside the pre-existing stress pattern will be found to be permanently preserved between two immobile vertical twinning walls.

Tuning seems to be similarly straightforward. To increase the spatial frequency of a pattern of stripes, it is merely necessary to regenerate the original stress pattern and to move whichever stress point lies near the vertical boundary so as to compress the area occupied by the zig-zag pattern.

As things stand, NPP crystals are plainly a long way from being practical devices with any kind of function. The regularity of the spacing of alternating wedges of twinned crystal has, however, been confirmed by the demonstration that they are capable of yielding sharp Bragg diffraction spots (with optical light, not Xrays). The interest of a laser which is also a tunable optical grating will require time to be fully appreciated. Intrinsic unprecedented collimation is presumably only one of the potential benefits.
John Maddox 